\title{
Polarization-tunable nonlinear absorption patterns from saturated absorption to reverse saturated absorption in anisotropic GeS flake and an application of all-optical switching
}

\author{
Hao Ouyang ${ }^{1,2,3 \dagger}$, Chenxi Zhang ${ }^{1 \dagger}$, Qirui $\mathrm{Liu}^{1 \dagger}$, Siyang $\mathrm{Hu}^{1}$, Jun Zhang ${ }^{4}$, Hao Hao ${ }^{4}$, Jie You ${ }^{5}$, \\ Xiang'ai Cheng ${ }^{1,2,3}$ and Tian Jiang ${ }^{1 *}$
}

\begin{abstract}
Due to the unique anisotropic chemical and physical properties, two-dimensional (2D) layered materials, such as IV-VI monochalcogenides with puckered honeycomb structure, have received considerable interest recently. Among the IV-VI layered MX (M = Ge, Sn; $X=S e, S)$ compounds, germanium sulfide $(\mathrm{GeS})$ stands out for its strongest anisotropic thermal conductivities and figure-of-merit values. Additionally, the layer-independent direct energy bands $\left(E_{\mathrm{g}} \sim 1.6 \mathrm{eV}, E_{1} \sim 2.1 \mathrm{eV}\right)$ of $\mathrm{GeS}$ flake provide excellent insights into further applications as visible photodetectors. Herein, the polarization-tunable nonlinear absorption (NA) patterns of GeS flake have been systematically investigated. Specifically, both the polarization-dependent Raman spectroscopy and the linear absorption (LA) spectroscopy were employed to characterize the lattice orientation and absorption edges of the 251-nm GeS flake. Considering the low damage threshold of $\mathrm{GeS}$ flake, the GeS/graphene heterostructure was fabricated to increase the threshold without changing the nonlinear properties of GeS. Our NA results demonstrated that a $600-\mathrm{nm}$ femtosecond laser with different polarizations would excite the saturated-absorption (SA) effect along armchair and reversesaturated-absorption (RSA) effect along zigzag in the $\mathrm{GeS} /$ graphene heterostructure. Moreover, the function of the polarization-based GeS/graphene heterostructure all-optical switch was experimentally verified. Notably, thanks to the polarization-dependent NA patterns (SA/RSA) of GeS, the
\end{abstract}

"ON" and "OFF" states of the all-optical switch can be accomplished by high and low transmittance states of continuous-wave laser $(532 \mathrm{~nm}, 80 \mathrm{nW})$, whose state can be controlled by the polarization of femtosecond switching laser $\left(600 \mathrm{~nm}, 35 \mathrm{fs}, 500 \mathrm{~Hz}, 12 \mathrm{GW} \mathrm{cm}{ }^{-2}\right)$. The ON/OFF ratio can achieve up to $17 \%$ by changing polarization, compared with the ratios of $3.0 \%$ by increasing the incident power of switching light in our experiment. The polarization-tunable absorption patterns introduced in this work open up real perspectives for the next-generation optoelectronic devices based on GeS/graphene heterostructure.

Keywords: polarization-tunable NA, I-scan, all-optical switch, modulated depth

\section{INTRODUCTION}

Two-dimensional (2D) layered materials, such as graphene [1-3], transition metal chalcogenides (TMDCs) [4-9], and topological insulators (TIs) [10-13], have received tremendous research attention in optical, electronic, and optoelectronic applications, due to their outstanding physical and chemical properties [11,14-17]. Most materials mentioned above are isotropic with polarization-independent optical and electronic properties. Differently, another group of materials with low lattice symmetry, such as black phosphorus (BP) [18-20], rhe-

${ }^{1}$ College of Advanced Interdisciplinary Studies, National University of Defense Technology, Changsha 410073, China

${ }^{2}$ State Key Laboratory of Pulsed Power Laser Technology, Changsha 410073 China

${ }^{3}$ Hunan Provincial Key Laboratory of High Energy Laser Technology, Changsha 410073, China

${ }^{4}$ College of Computer, National University of Defense Technology, Changsha 410073, China

${ }^{5}$ National Innovation Institute of Defense Technology Beijing 100010, China

† These authors contributed equally to this work.

* Corresponding author (email: tjiang@nudt.edu.cn) 
nium disulfide $\left(\operatorname{ReS}_{2}\right)$ [21,22], and gallium telluride (GaTe) [23], have recently attracted attention for their polarization-dependent thermal conductivity [24], optical absorption [14], and third harmonic generation (THG) [25]. As for anisotropic materials, there are mainly two typical types of in-plane atom structures [26]: the first one is $\mathrm{ReS}_{2}$-like TMDC, which consists of atoms distributed in disordered trigonal (1T) structure [14]; the other one is a material represented by black phosphorus (BP), such as four-six-enes [27], which shows a puckered honeycomb structure. In contrast to other hexagonal (2H) TMDCs, the origin of anisotropy in $1 \mathrm{~T}$ structure of $\mathrm{ReS}_{2}$ is caused by the additional $d$ valence electron of the Re atoms. It is the electron that accounts for zigzag Re chain parallel to the $b$-axis of the crystal [14]. Therefore, the symmetry of $\mathrm{ReS}_{2}$ is drastically reduced. This low symmetry prompts $\mathrm{ReS}_{2}$ to be one of the most promising candidates for polarization-sensitive detectors with proper mobility $\left(\sim 40 \mathrm{~cm}^{2} \mathrm{~V}^{-1} \mathrm{~s}^{-1}\right)$ and ON/OFF ratio $\left(\sim 10^{5}\right)$ [22]. Another typical anisotropic material is BP. Its puckered honeycomb structure makes it stand out. Precisely, the thermal conductivities of BP along armchair and zigzag are $~ 20$ and $40 \mathrm{~W} \mathrm{~m}^{-1} \mathrm{~K}^{-1}$ on thick film [24]. In addition, the ratio of conductivities $\sigma_{x} / \sigma_{y}$ and ratio of mobilities $\mu_{x} / \mu_{y}$ are both 1.5 [28]. Apart from the anisotropy properties, BP exhibits alternative distinguished features of the thickness-dependent bandgap, which can be tailored from $0.3 \mathrm{eV}$ (bulk) to $1.5 \mathrm{eV}$ (monolayer), as well as the layerdependent charge-carrier mobility that reaches $\sim 1000 \mathrm{~cm}^{2} \mathrm{~V}^{-1} \mathrm{~s}^{-1}$ at room temperature [29] and the extremely high ON/OFF ratio of $>10^{5}$ [28].

Similar to the puckered layer structure of BP, the group IV-VI compounds (GeS [30], GeSe [31,32], SnS [33], and SnSe [34]) have shown great application possibilities in photodetectors, photovoltaic, piezoelectric, and thermoelectric devices, due to their earth abundance, environmental compatibility, less toxicity, and anisotropic properties [35-37]. Among these 2D-layered monochalcogenides, the monolayer GeS has the largest ratio (3.38) of lattice thermal conductivities along zigzag and armchair directions at $300 \mathrm{~K}$ [37]. Moreover, GeS also possesses the largest ratio of figure-of-merit $Z T$ $\left(Z T_{\text {armchair }} / Z T_{\text {zigzag }}\right)$, which is $1.434(1.85 / 1.29)$, compared with 1.069 of SnSe (2.63/2.46), 1.074 of SnS (1.88/1.75), and 1.15 of GeSe (1.99/1.73) [38]. On the other hand, multilayer $\mathrm{GeS}$ is an anisotropic band-edge transition semiconductor with a direct-bandgap varying from 1.60 to $1.65 \mathrm{eV}$ [39] and its electron mobility is $3680 \mathrm{~cm}^{2} \mathrm{~V}^{-1} \mathrm{~s}^{-1}$, which is much higher than that of $\mathrm{MoS}_{2}$ and BP [40]. Apart from high carrier mobility of GeS, other properties, such as stain tunable energy band effect, substantial excitonic effect, spin-orbit interaction, and good stability, have also been investigated [34,40-44]. Owing to a number of excellent physical properties of GeS, different experimental techniques are thoroughly utilized to characterize its optical anisotropic response. For instance, the photoluminescence (PL) was used to characterize the transition of GeS flakes near their band edges [45]. The polarization-dependent linear transmittance spectroscopy and PL effect were both properly studied to verify the anisotropic energy bands of GeS [39]. In the meantime, the Raman-scattering spectroscopy was used to identify the crystal orientation of $2 \mathrm{D}$ anisotropic materials (e.g., GeS and SnSe) [26,27,34,46].

Apart from the outstanding optical and chemical properties, the application prospects of GeS are also promising. Thanks to former studies [43], there are some important applications in electronic and optoelectronic devices based on GeS solar cells and GeS-field-effect transistors (GeS-FETs). It is worth noting that there are several important performance factors for GeS-FETs, including the photoresponsivity $\left(\sim 206 \mathrm{~A} \mathrm{~W}^{-1}\right)$, the external quantum efficiency (EQE, $\left.\sim 4.0 \times 10^{4} \%\right)$, the specific detectivity $D^{*}\left(\sim 2.35 \times 10^{13}\right.$ jones $)$, and the ON/OFF ratio $\left(\sim 10^{14}\right)[39,47,48]$. To this end, the experimental exploration of tunable optical and electronic properties in layered GeS is mainly focused on stain $[44,49]$, defect/ doping [50], heterostructure [51-53] in experiments, with the theoretical calculations based on density functional theory (DFT) calculation [52,54]. However, different from most TMDCs, whose energy absorption could be controlled by changing their thickness or using complex chemical doping $[4,6]$, GeS flakes naturally possess polarization-dependent absorption edges. So far, saturated absorption (SA)-based all-optical switching without polarization has been broadly researched, while polarization-tunable all-optical switching has not. On account of this specialness of GeS, it is meaningful to take advantage of the polarization-tunable nonlinear optical absorption responses (SA/RSA, RSA=reverse saturated absorption) as all-optical switching.

In this work, we presented a comprehensive investigation of the in-plane anisotropic optical properties of a $251 \mathrm{~nm}$ layered GeS. Specifically, the measurements of polarization-dependent Raman scatting, linear absorption (LA) spectra, and nonlinear optical absorption were performed on the layered GeS mechanically exfoliated from the bulk GeS crystal. One significant finding from Raman scatting spectra is that the armchair and zigzag directions of the GeS flakes can be characterized. Anisotropic LA 
spectra were utilized to determine the polarization-dependent absorption edges, which are $2.020 \mathrm{eV}$ along $\operatorname{armchair}\left(E_{\mathrm{g}, \|}, \theta=0^{\circ}\right)$ and $2.116 \mathrm{eV}$ along zigzag $\left(E_{\mathrm{g}, \perp}\right.$, $\left.\theta=90^{\circ}\right)$. Moreover, the absorption edges along different polarizations are within them, obeying the Malus law. Furthermore, the polarization-dependent I-scan technique was performed to measure nonlinear absorption (NA) properties of the GeS flakes under a series of incident light power. Interestingly, a $600 \mathrm{~nm}(2.067 \mathrm{eV})$ femtosecond laser can excite both single-photon absorption (1PA) and two-photon absorption (2PA) under different polarized directions. Similar to the 1PA, 2PA is also an interband transition in which two photons with energy of $2 \hbar \omega$ are absorbed simultaneously, resulting in an electron jumping from the valence band to the conduction band. Herein, 1PA and 2PA mainly dominate the SA and RSA responses, respectively. The modulation depths of GeS along the armchair and zigzag directions are $\sim 1.5 \%$ and $\sim-4 \%$, respectively, from SA state to RSA state $[4,6,55]$. Equally remarkable, a model for the GeS/graphene heterostructure-based all-optical switch, whose "ON" and "OFF" states can be achieved by altering the transmittance states of continuous wavelength (CW) signal light, has been experimentally studied and then verified.

\section{EXPERIMENTAL SECTION}

\section{Sample preparation and characterization}

The GeS flakes were mechanically exfoliated from bulk GeS crystal onto a quartz substrate. The thickness and roughness of the GeS flake were characterized by atomic force microscopy (AFM). Moreover, since the GeS flake tends to be damaged under a large illuminating light power, a few-layer graphene was firstly mechanically exfoliated from graphene crystal onto silicon $(\mathrm{Si})$ substrate and then was dryly transferred upon GeS flake.

\section{Polarization-dependent Raman spectroscopy}

To characterize the lattice orientation of the GeS flake, a polarization-dependent Raman measurement was employed. In the Raman microscopy experiment setup, a $532 \mathrm{~nm}$ linear polarized CW laser with low-power ( 100 $\mathrm{nW}$ ) was coupled to a confocal microscope (LEICA DM $2700 \mathrm{M})$ to excite the anisotropic sample with a $100 \times$ objective. The polarization-dependent Raman spectra of GeS were detected by the spectrometer (ANDOR SR500i-B1-R), whose analyzer was parallel to the incident light polarization; thus, a parallel collection configuration was achieved. Therefore, the polarization-dependent Raman spectra of GeS flake were obtained by rotating the polarizer and the analyzer simultaneously every $15^{\circ}$, from $0^{\circ}$ to $180^{\circ}$. To ensure the same detection point in each test, the position of the GeS sample was carefully adjusted, and the microscope focus was optimized to reduce the errors.

\section{Anisotropic LA spectroscopy}

The polarization-dependent LA spectra were measured using a commercial micro-spectrometer (Micro, ideaoptics, China). In this setup, the illuminating light in the microscope is a halogen lamp with a wavelength range from 350 to $2500 \mathrm{~nm}$, accompanied by an Olympus MPlanFL N 100× objective serving as the focusing unit. Simultaneous measurements of the reflection $(R)$ and transmission $(T)$ were then performed. The peak power was low enough to avoid nonlinear optical effects. Thus, the LA rate $(1-R-T)$ at different polarization angles with a $15^{\circ}$ increment each time was obtained.

\section{Nonlinear optical measurements}

An open-aperture micro-I-scan system was constructed to explore the anisotropic NA of GeS flake. In the system, a broad-range wavelength objective (Mitutoyo 50×, NA = $0.65,400-1700 \mathrm{~nm}$ ) was used to focus the incident light into a small spot. The light sources $(600 \mathrm{~nm}, 1 \mathrm{kHz})$ of the I-scan were generated from an optical parametric amplifier (TOPAS) with the output of a Ti:sapphire laser system laser (Spectra-Physics, $35 \mathrm{fs}, 800 \mathrm{~nm}, 1 \mathrm{kHz}$ ). A continuously adjustable neutral density filter (Thorlabs, NDL-10C-4) was adopted to modulate the laser intensity, allowing for the I-scan measurement. After that, the reputation frequency of $600 \mathrm{~nm}$ light was modulated from $1 \mathrm{kHz}$ to $500 \mathrm{~Hz}$ by a Chopper (Thorlabs, MC2000B-EC). Next, a half-wave plate and a Gran prism were employed to adjust the polarization of the incident light. An imaging camera and a confocal microscope (LEICA DM $2700 \mathrm{M}$ ) were used to build the video microscope system. The femtosecond laser was focused on the sample into a spot with a diameter of $3 \mu \mathrm{m}$. Additionally, two Si-detectors with dual-channel lock-in amplifiers (Sine Scientific Instrument, OE1022D), one detector for monitoring the reference light, and the other for collecting the light through the sample, were utilized to measure the nonlinear transmittance more accurately. By adjusting the polarization of the incident light, the polarization-dependent nonlinear transmittance was obtained.

\section{All-optical switching experiments}

Switching light $\left(600 \mathrm{~nm}, 35 \mathrm{fs}, 500 \mathrm{~Hz}, 12 \mathrm{GW} \mathrm{cm}^{-2}\right)$ with a strong intensity was used to modulate the much weaker 
signal light $(532 \mathrm{~nm}, \mathrm{CW}, 80 \mathrm{nW})$. A coaxial $600 \mathrm{~nm}$ fs laser and a $532 \mathrm{~nm} \mathrm{CW}$ laser beam were tightly co-focused by an objective (Mitutoyo $50 \times$ ) with parallel polarization. A half-wave plate and a Gran prism were used to change the polarization of the incident light. Using an optical bandpass filter, only the signal light at $532 \mathrm{~nm}$ was collected by the detectors.

\section{RESULTS AND DISCUSSION}

Preparation and lattice orientation characterization of GeS Layered $\mathrm{GeS}$ has a distorted orthorhombic structure (space group $P c m n-D_{2 h}^{16}$ ) $[37,45]$, in which the $S$ atoms of
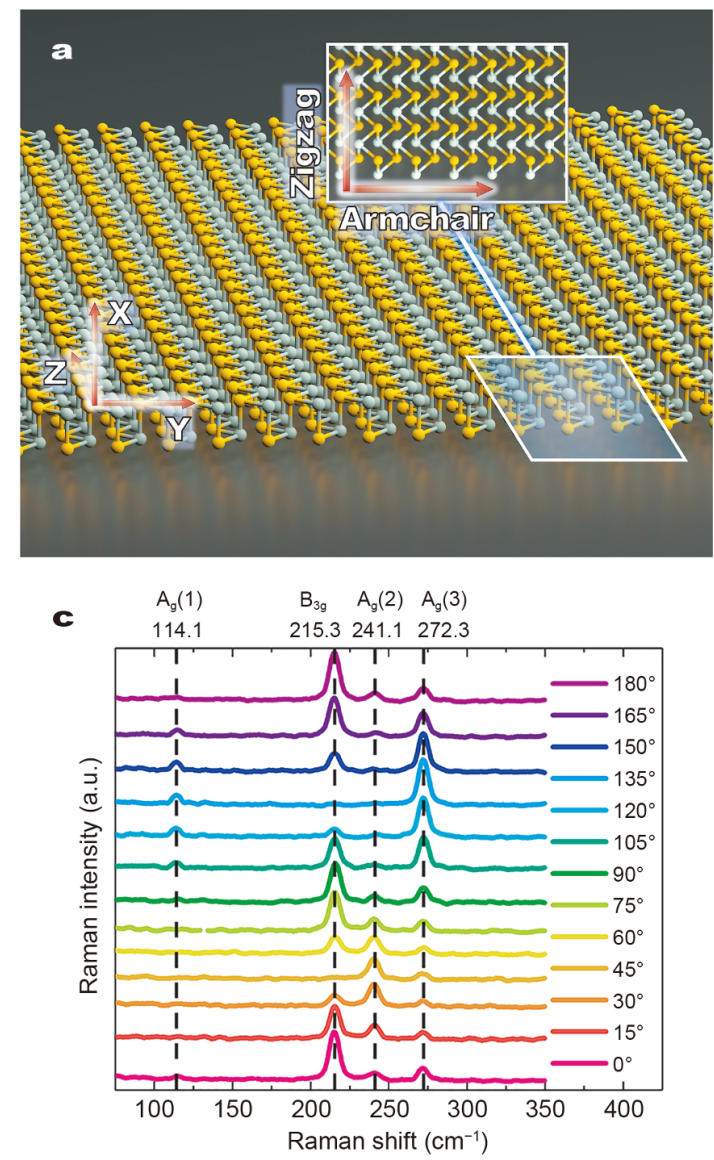

each layer are covalently bonded to three adjacent Ge atoms to form a puckered honeycomb network in the $Y-Z$ plane, as shown in Fig. 1a. Due to the weak $X$-direction van der Waals force, the adjacent GeS layers are able to be exfoliated from each other easily. Here, the GeS flake was prepared by the mechanical-exfoliation method and then transferred onto a quartz substrate. The optical image and AFM image of the layered GeS flake are shown in Fig. 1 b. In this regard, the GeS flake with a $61 \mathrm{~nm}$ length and a $31 \mathrm{~nm}$ width satisfies our experimental expectation. The armchair and zigzag directions along the border of the GeS flake are characterized via the angle-dependent Raman spectra. In addition, the insert AFM image in Fig. 1b shows the height profile of the layered GeS, whose
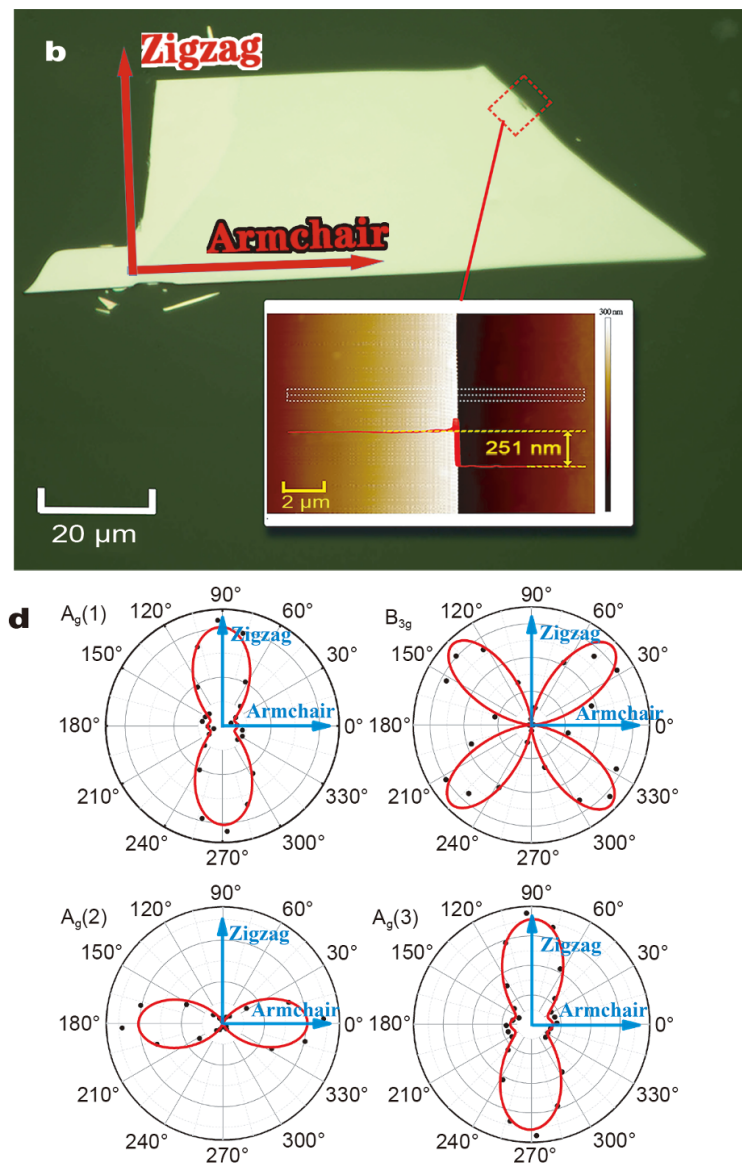

Figure 1 Atomic structure diagram and polarization-dependent Raman spectra of GeS flake. (a) Schematics of the layered GeS crystal structure: orthogonal view and the top view (inset), with the armchair $(Y)$ and zigzag $(Z)$ directions marked by red arrows. The yellow and grey particles represent the Ge and S atoms, respectively. (b) Optical and AFM (inset) images of the GeS flake. The red arrows indicate the armchair and zigzag directions characterized via Raman spectroscopy. (c) The polarization-dependent Raman spectra of GeS flake in the parallel polarization configuration with a $532 \mathrm{~nm}$ excitation. The curves with different colors represent the Raman spectra at different light incident angles. The sample was rotated from $0^{\circ}$ to $180^{\circ}$ with a step size of $15^{\circ}$ (in order to make the polar diagram better looking, data from $180^{\circ}$ to $360^{\circ}$ were the repetition of that from $0^{\circ}$ to $180^{\circ}$ ). The four typical Raman peaks $\left(114.1,215.3,241.1\right.$, and $272.3 \mathrm{~cm}^{-1}$ ) are associated with the $A_{g}(1), B_{3 g}, A_{g}(2)$ and $A_{g}(3)$ modes, respectively. (d) Intensities of angular-dependent peaks of the four Raman modes. The black dots are the experimental results, while the red curves show the fitting results obtained using Equation (2) for $A_{g}(1), A_{g}(2), A_{g}(3)$ modes and Equation (3) for $B_{3 g}$ mode. 
thickness is $\sim 251 \mathrm{~nm}$.

Raman-scattering spectroscopy is an efficient and widely used tool for characterizing the lattice structure and the lattice vibrations (photons) of layered materials [56]. Under the polarization-dependent light excitation, the angle-dependent Raman spectroscopy can analyze the crystal orientation in parallel configurations [32,34,45]. It can be seen from Fig. 1c that four typical Raman peaks of $\mathrm{GeS}$ at 114.1, 215.3, 241.1, and $272.3 \mathrm{~cm}^{-1}$ can be assigned to four phonon vibration modes with $A_{g}(1), B_{3 g}, A_{g}(2)$, and $A_{g}(3)$ symmetry [45]. To further determine the crystal orientation, the angle-dependent Raman intensities of GeS were obtained by integrating the intensities of $A_{g}$ and $B_{3 g}$ modes. The intensities of the four typical Raman modes are shown in polar plots (Fig. 1d). Particularly, $0^{\circ}$ in the polar plots corresponds to the direction of armchair, while $90^{\circ}$ is parallel to the direction of zigzag. Additionally, $\mathrm{A}_{\mathrm{g}}$ modes show an obvious spindle shape with a 180-degree rotational symmetry, whereas $B_{3 g}$ mode has a period of 90-degree like dualspindle shape, as displayed in Fig. $1 \mathrm{~d}$. The above phenomenon could be well explained by the classical Placzek model. The Raman intensity $I$ is proportional to $\left|\overrightarrow{\boldsymbol{e}}_{\mathrm{i}} \cdot \widetilde{\boldsymbol{R}} \cdot \overrightarrow{\boldsymbol{e}}_{\mathrm{s}}^{T}\right|^{2}$, where $\widetilde{\boldsymbol{R}}$ is the Raman tensor for the Raman active mode, $\overrightarrow{\boldsymbol{e}}_{\mathrm{i}}$ and $\overrightarrow{\boldsymbol{e}}_{\mathrm{s}}^{T}$ are the unitary electric polarization vectors of the incident and scattered light, respectively. Under our test conditions, $\overrightarrow{\boldsymbol{e}}_{\mathrm{i}}$ and $\overrightarrow{\boldsymbol{e}}_{\mathrm{s}}$ are in the $Y-Z$ plane. Precisely, the vector $\overrightarrow{\boldsymbol{e}}_{\mathrm{i}}$ is described as $(0, \cos \theta, \sin \theta)$, where $\theta$ is the polarization angle of incident laser related to the armchair direction. $\overrightarrow{\boldsymbol{e}}_{\mathrm{s}}$ is defined by $(0, \cos \theta, \sin \theta)$ for the scattered light in the parallel polarization configuration. Since GeS is in the $D_{2 h}$ space group, the Raman tensors of the $A_{g}$ and $B_{3 g}$ modes can be given by [34]

$\widetilde{\boldsymbol{R}}_{\mathrm{Ag}_{\mathrm{g}}}=\left(\begin{array}{ccc}a & 0 & 0 \\ 0 & b & 0 \\ 0 & 0 & c\end{array}\right), \quad \widetilde{\boldsymbol{R}}_{\mathrm{B}_{3 g}}=\left(\begin{array}{ccc}0 & 0 & 0 \\ 0 & 0 & f \\ 0 & f & 0\end{array}\right)$,

where, $a, b, c$, and $f$ are the Raman-tensor parameters. Therefore, the angular-dependent Raman intensity can be expressed as follows [57]:

$I_{\mathrm{A}_{\mathrm{g}}}^{\|} \propto\left(|b| \cos ^{2} \theta+|c| \cos \varphi_{b c} \sin ^{2} \theta\right)^{2}$

$+|c|^{2} \sin ^{2} \varphi_{b c} \sin ^{4} \theta$

$I_{\mathrm{B}_{3 \mathrm{~g}}}^{\|} \propto(2|f| \sin \theta \cos \theta)^{2}=|f|^{2} \sin ^{2} 2 \theta$.

It is worth noting that in the case of absorptive materials, the four Raman tensor elements $(a, b, c$ and $f)$ are complex numbers. Here, $\varphi_{b c}$ represents the phase difference $\varphi_{b}-\varphi_{c}$ between $b$ and $c$, and $\varphi_{f}$ vanishes due to the square modulus of the Raman intensity expressions. Using the Equations (2) and (3) to fit the angle-dependent intensities of $\mathrm{A}_{\mathrm{g}}$ and $\mathrm{B}_{3 \mathrm{~g}}$ modes shown as the red lines in Fig. 1d, we determined the armchair and zigzag directions of our GeS flake in the optical image (marked with red arrows in Fig. 1b).

\section{Anisotropic LA spectra of GeS}

The LA spectra were recorded to estimate the band gaps and absorption edges of $2 \mathrm{D}$ materials [58]. Next, the transmission and reflection lights were collected by spectrograph in different modes. In this context, the Tauc model was applied to analyze the LA of GeS and their lowest excitonic state $E_{\mathrm{g}}$ :

$\alpha h v=A\left(h v-E_{\mathrm{g}}\right)^{n}$

where, $h$ is Plank constant, $v$ is the photon frequency, and $A$ is a constant connected to the matrix element. The constant parameter $n$, which is related to the material transition type, is 0.5 for layered GeS due to its direct transition [59]. However, owing to the small difference between $E_{1, \|}$ and $E_{1, \perp}$ of $\mathrm{GeS}$, more in-depth exploration of the angular dependence absorption edges of GeS could be acquired by derivative transmittance spectra method [39].

In our experiment, the linear transmittance spectra $T(\lambda)$ and linear reflectance spectra $R(\lambda)$ were collected directly by a spectrograph. Then, linear absorption $A(\lambda)$ was calculated according to the following relation:

$A(\lambda)=1-T(\lambda)-R(\lambda)$.

Then, the LA rate changed with wavelength in Fig. 2a covering the range of 500-640 nm. Here, "wavelength" was chosen instead of "energy" as $X$-axis due to better selecting out the wavelength of nonlinear exciting laser. Seen from the figure, there are two obvious flatforms: below $560 \mathrm{~nm}$ and upper to $620 \mathrm{~nm}$. In other words, we focused on the range of the wavelength from 560 to $620 \mathrm{~nm}$. In order to select the proper wavelength to excite the anisotropic NA, polarization-dependent linear absorptance was used as an important reference frame. In addition, the band edges along armchair and zigzag are $2.02 \mathrm{eV}(614 \mathrm{~nm})$ and $2.12 \mathrm{eV}(586 \mathrm{~nm})$, respectively, which will be determined later. Thus, it is concluded that $600 \mathrm{~nm}(2.06 \mathrm{eV})$ is the best choice for studying the polarization-tunable absorption effects in GeS.

The determination of band edges was carried out as follows (Fig. 2b). From the figure, the derivative trans- 
mittance spectra were utilized to determine the absorption edges $E_{1, \|}$ and $E_{1, \perp}$ of GeS, via a method similar to that in the literature [39]. For our research, two typical angles of $0^{\circ}$ and $90^{\circ}$ are acquired after analyzing different polarization directions for these derivative curves. It is obvious that two valleys with the largest intensities are located at 586 and $614 \mathrm{~nm}$ and at the polarization of $90^{\circ}$ (zigzag) and $0^{\circ}$ (armchair), respectively. Notably, the $E_{1, \|}$ feature is the most prominent one near $E_{1, \|} \| b$ (armchair, $0^{\circ}$ ) polarization at $2.02 \mathrm{eV}$, whereas $E_{1, \perp}$ is sharply increased close to the $E_{1, \perp} \perp b$ (zigzag, $90^{\circ}$ ) polarization at $2.12 \mathrm{eV}$. The intensities of the two valleys are gradually changing between the armchair and zigzag polarizations. Here, the polarization-dependent equivalent energy bands are described as $E_{1}(\theta)=E_{1}(0)+\Delta \cdot|\sin \theta|$, where $E_{1}(0)$ is the band edge of $E_{1, \|}$, and $\Delta$ is the amplitude of energy variation. Based on the fitting results, the $E_{1, \|}$ value is determined to be $2.02 \mathrm{eV}$ with a $\Delta$ of $0.1 \mathrm{eV}$.

In order to analyze the two changing intensities of valleys and their relation with the increase of the angle, the curves was fitted by a double-peak fitting function. Then, the intensities $I_{586}$ and $I_{614}$ were obtained; the plot with the formula $\left(I_{586}-I_{614}\right) /\left(I_{586}+I_{614}\right)$ as ordinate and polarization angle as abscissa is displayed in Fig. 2c. A ratio of $\left(I_{586}-I_{614}\right) /\left(I_{586}+I_{614}\right)$ below 0 indicates the band edge of $614 \mathrm{~nm}$ taking the main role, which will tend to induce 1PA effect at a large incident power of $600 \mathrm{~nm}$ laser, whereas a ratio above 0 indicates that band edge of
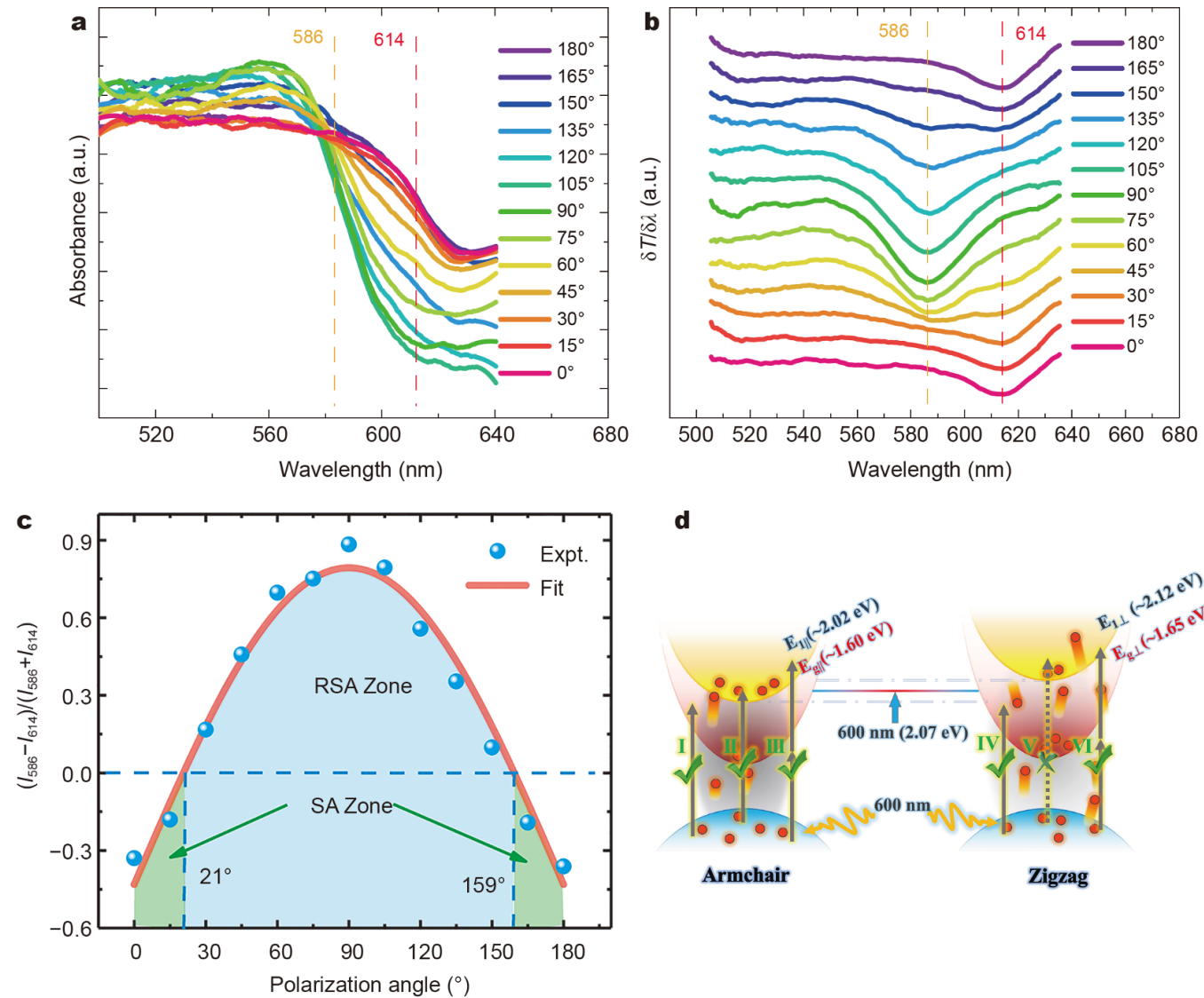

d

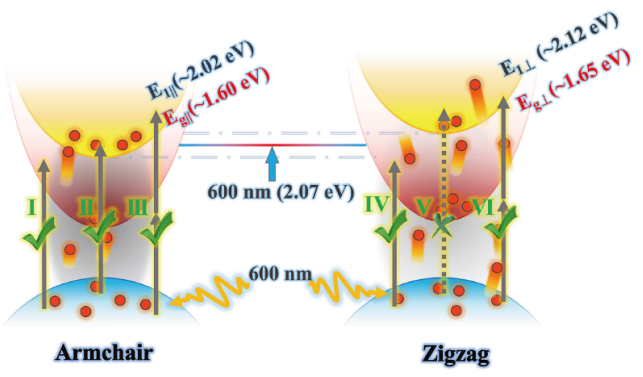

Figure 2 Energy edge characterization and polarization-dependent NA mechanism of GeS. (a) Polarization-dependent LA spectra of GeS from $0^{\circ}$ to $180^{\circ}$. There are two target dotted lines in yellow $(586 \mathrm{~nm})$ and red $(614 \mathrm{~nm})$, representing energy edges. (b) Derivative transmittance spectra ranging from $0^{\circ}$ to $180^{\circ}$. There are two most evident valleys at the wavelength of $586 \mathrm{~nm}$ and $614 \mathrm{~nm}$ under $0^{\circ}$ (armchair) and $90^{\circ}$ (zigzag) direction. Between armchair and zigzag directions, these two peaks exhibit the relationship of competition. (c) Ratio relationship of intensities between 586 and $614 \mathrm{~nm}$ peaks. Double-peak Gaussian fitting curve is used to fit each line; the intensity peaks of 586 and $614 \mathrm{~nm}$ are obtained and named $I_{586}$ and $I_{614}$, respectively. The experimental and fit results are expressed in blue dots and red lines, respectively. Besides, zeros of the fit curve are at $21^{\circ}$ and $159^{\circ}$. The blue area represents that the band edges of $2.12 \mathrm{eV}(586 \mathrm{~nm})$ are prevailing and correspond to the RSA Zone at the excitation of $600 \mathrm{~nm}$. Then, the green area is defined as SA Zone. (d) Diagrammatic sketch of the two types of NA mechanisms in GeS along armchair and zigzag. Type I, II, and III absorptions can co-exist along armchair, which is in the SA Zone. However, only type IV and type VI exist along zigzag. 
$586 \mathrm{~nm}$ is easy to dominate, causing the excitation of $2 \mathrm{PA}$ effect. Additionally, two zeros are observed at $21^{\circ}$ and $159^{\circ}$ for the same curve in Fig. 2c. Thus, the 1PA effect dominates in the two green zones (polarization angles from $0^{\circ}$ to $21^{\circ}$ and from $159^{\circ}$ to $180^{\circ}$ ), while the $2 \mathrm{PA}$ effect plays the main role in the blue region (polarization angle between $21^{\circ}$ and $159^{\circ}$ ). The origin of polarizationdependent absorption edges and NA mechanisms of GeS can be further explained by the Fermi's golden rule. The transition rate, defined as the probability of a transition per unit time, is mathematically described as [57]

$\Gamma_{i \rightarrow f}=\frac{2 \pi}{\hbar}\left|\left\langle f\left|H_{\mathrm{op}}\right| i\right\rangle\right|^{2} \rho_{f}$,

where, $\rho_{f}$ is the density of the final $(f)$ states, and $\left\langle f\left|H_{\mathrm{op}}\right| i\right\rangle$ is the electron-phonon matrix element of the perturbation $H_{\mathrm{op}}$ between the final and initial (i) states. The matrix element can be calculated within the dipole approximation, with the optical transition from state $i$ to $f$ [60], which can be expressed as

$\left|\left\langle f\left|H_{\mathrm{op}}\right| i\right\rangle\right| \propto P \cdot D_{f i}$,

where, $P$ is the polarization vector of the incident light, and $D_{f i}$ is the dipole vector, defined as $D_{f i}=\langle f|\nabla| i\rangle$. Moreover, another crucial factor is the optical absorption probability, which can be described by the absorption coefficient $\alpha$ :

$\alpha\left(E_{\mathrm{L}}\right) \propto \sum_{f, i}\left|\left\langle f\left|H_{\mathrm{op}}\right| i\right\rangle\right|^{2} \delta\left(E_{f}-E_{i}-E_{L}\right)$,

where, $E_{\mathrm{L}}$ is the incident photon energy, and $E_{i}\left(E_{f}\right)$ is the band energy of the electronic state $i(f)$. According to Equations (7) and (8), the absorption coefficient $\alpha$ is proportional to the square of the inner product of the polarization vector $P$ and the dipole vector $D_{f i}$. With the change of incident light polarization, the energy bands associated with electron transition are also different. Therefore, based on the selection rule, the polarizationdependent band edges would gradually vary from $2.02 \mathrm{eV}$ at armchair $\left(0^{\circ}\right)$ direction to $2.12 \mathrm{eV}$ at zigzag $\left(90^{\circ}\right)$ direction.

Although the angle-dependent band energies and their origins are clarified, it is still necessary to understand the optical processes in GeS flake under a $600 \mathrm{~nm}$ femtosecond light excitation. According to previous reports $[39,41,61]$, GeS owns several distinct excitonic states, such as $E_{\mathrm{g}}$ at $\sim 1.62 \mathrm{eV}$ and the first prominent absorption edge $\left(E_{1}\right)$ at $\sim 2.1 \mathrm{eV}$. In order to describe the polarizationdependent energy bands of $251 \mathrm{~nm}$ GeS flake clearly, we reported the schematic illustration of absorption energies along armchair and zigzag in Fig. 2d. Along armchair (// direction), $E_{\mathrm{g}, \|}$ and $E_{1, \|}$ are 1.60 and $2.02 \mathrm{eV}$, respectively, while these two factors turn to be 1.65 and $2.12 \mathrm{eV}$ along zigzag polarization ( $\perp$ direction). In the following nonlinear I-scan experiments, we mainly focused on the polarization-dependent first absorption energy band $\left(E_{1}\right)$. Thus, a polarized femtosecond laser with $2.06 \mathrm{eV}$ photon energy was chosen to excite NA. When the polarization direction of the $600 \mathrm{~nm}(2.06 \mathrm{eV})$ laser is parallel to the armchair, as shown in Fig. 2d, the absorption type I, II, and III can co-exist. More specifically, Type I indicates the process that an electron from valance band maximum (VBM) absorbs a single photon and transmits to $E_{\mathrm{g}, \|}$. Type II describes the process that an electron from VBM absorbs a single photon and transmits to $E_{1, \|}$. Type III specifies the process that an electron from VBM absorbs two photons and transmits to $E_{1, \|}$. Another state is the laser polarization direction perpendicular to armchair. In this situation, the transition types IV and VI can exist, while type $\mathrm{V}$ is blocked since it does not meet the selection rule. It is noticeable that types IV, V, and VI correspond to type I, II, and III, respectively.

\section{Polarization-tunable nonlinear optical absorption of GeS} Another significant property of $\mathrm{GeS}$ to be explored is the anisotropic NA. Considering that GeS can be easily damaged under a high incident light intensity of $15 \mathrm{GW} \mathrm{cm}^{-2}$ with a low thermal conductivity (6.38 $\mathrm{W} \mathrm{m}^{-1} \mathrm{~K}^{-1}$ ) [37] and a low melting point $\left(615^{\circ} \mathrm{C}\right)$, we transferred a few-layer graphene onto the GeS nanoflake in order to build the GeS/graphene heterostructure as depicted in Fig. 3. This approach exploits the significantly high thermal conductivity of graphene (up to $5300 \mathrm{~W} \mathrm{~m}^{-1} \mathrm{~K}^{-1}$ ) [62] to enhance the tolerance of the incident light intensity. Traditionally, the Z-scan technique [63-65] is usually adopted to investigate both the nonlinear refraction (closed-aperture setup) and the NA properties (open-aperture setup); therefore, the sample needs to be fabricated in large scale such as continuous membrane by chemical vapor deposition (CVD) or molecular beam epitaxy (MBE) method. However, in the Iscan system, it is possible to adjust the optical attenuator to obtain the continuously varying incident intensity, in which the spot size of the incident beam is constant for few microns. Therefore, the polarization-dependent micro I-scan technique was employed to investigate the anisotropic NA of the GeS/graphene heterostructure. More information about the I-scan system can be found 


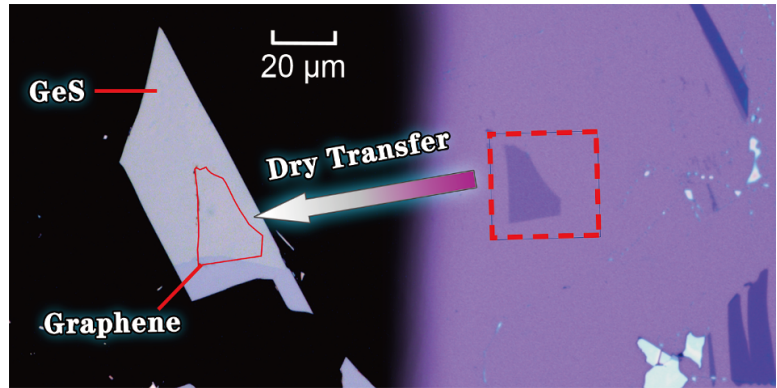

Figure 3 The optical images before and after dryly transfer. The GeS/ graphene heterostructure on quartz (left figure) and the graphene on $\mathrm{Si}$ (before the dry-transfer process, right figure).

in the EXPERIMENTAL SECTION.

In our experiment setup, $\theta$ was the polarization angle of the incident light $\left(600 \mathrm{~nm}, 35 \mathrm{fs}, 500 \mathrm{~Hz}, 19.58 \mathrm{GW} \mathrm{cm}^{-2}\right.$ ) to the armchair direction, which changed from $0^{\circ}$ to $180^{\circ}$ in $15^{\circ}$ step, and the step reduced to $5^{\circ}$ near armchair polarization. As illustrated in Fig. 4a, the normalized transmittance (NT) undergoes a rapid increase with the initial increase in the $0^{\circ}$ incident light intensity and then tends to remain constant when the incident beam is sufficiently strong, indicating SA response in the heterostructure. However, a dramatic difference appears in the $90^{\circ}$ incident light case. It can be seen from Fig. $4 \mathrm{~b}$ that
NT decreases as the intensity of the $90^{\circ}$ incident light increases, which implies RSA response. Based on the Beer-Lambert law, the attenuation of light passing through an optical medium over a propagation distance $z$ can be described as:

$\frac{d I}{d z}=-\alpha(I) I$,

where $\alpha(I)$ is the intensity-dependent absorption coefficient. With the NA response of our GeS/graphene heterostructure switching from SA to RSA, the saturation of both the 1PA and the 2PA needs to be considered. Similar to the $1 \mathrm{PA}, 2 \mathrm{PA}$ is also an interband transition in which two photons with energy of $2 \hbar \omega$ are absorbed simultaneously, resulting in an electron jumping from the valence band to the conduction band. During these two transitions, when excited by a sufficiently high incident intensity, denoted as the saturation intensity, atoms in the ground state of the heterostructure can be excited into an upper energy state at such a rate that there is insufficient time for the atoms to decay back until all the excited states are filled, leading to the saturation effect. Thus, the absorption coefficient $\alpha(I)$ is given by:

$\alpha(I)=\alpha+\beta I=\frac{\alpha_{0}}{1+I / I_{\mathrm{S}_{1}}}+\frac{\beta_{0} I}{1+\left(I / I_{\mathrm{S}_{2}}\right)^{2}}$,
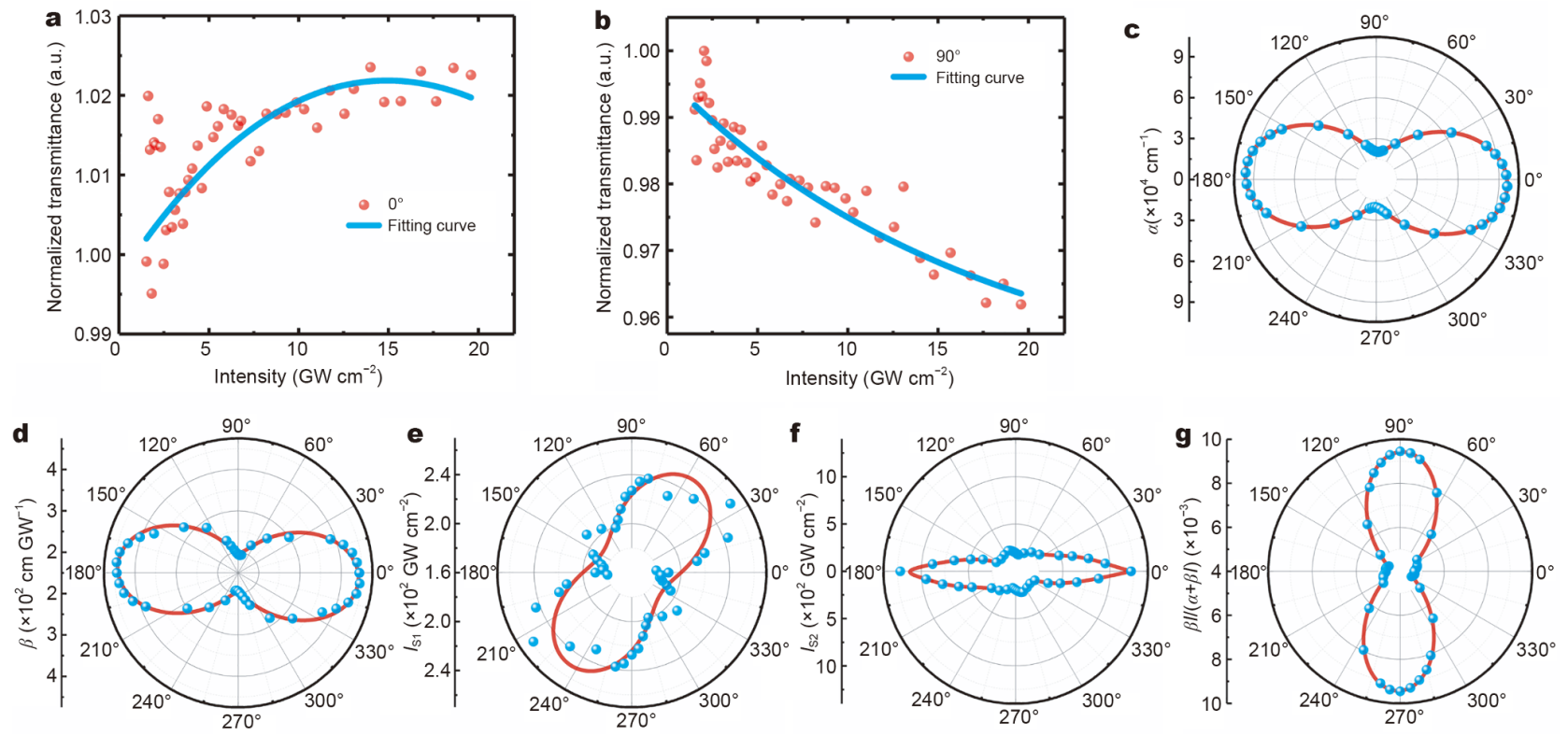

Figure 4 I-scan results of normalized transmittance with the polarization of incident light (a) parallel and (b) perpendicular to the armchair direction. The red dots are the experimental data, and the blue curves stand for the fittings. (c-f) The variation of fitting parameters (blue dots) based on Equation (3) versus polarization angle to the armchair direction in polar coordinates. (c) LA coefficient. (d) 2PA coefficient. (e) Saturation intensity of LA. (f) Saturation intensity of 2PA. (g) The dependence of $k$ on polarization angle with $k=\beta I /(\alpha+\beta I)$ representing the proportion of $2 \mathrm{PA}$ in the overall NA. 
where $\alpha=\alpha_{0} /\left(1+I / I_{\mathrm{S}_{1}}\right), \beta=\beta_{0} I /\left(1+I^{2} / I_{\mathrm{S}_{2}}{ }^{2}\right), \alpha_{0}$ and $\beta_{0}$ are the LA and $2 \mathrm{PA}$ coefficients, respectively. $I_{\mathrm{S}_{1}}$ and $I_{\mathrm{S}_{2}}$ are their saturation intensities, respectively. Derived from Equations (1) and (2), the intensity-dependent transmittance $T(I)$ after passing through the sample with a thickness of $L$ is expressed as [57]

$T(I)=\exp \left(-\left(\frac{\alpha_{0}}{1+I / I_{\mathrm{S}_{1}}}+\frac{\beta_{0} I}{1+\left(I / I_{\mathrm{S}_{2}}\right)^{2}}\right) L\right)$.

The values of $\alpha_{0}, \beta_{0}, I_{\mathrm{S}_{1}}, I_{\mathrm{S}_{2}}$ are extracted by fitting the I-scan results with Equation (11), and then drawn into polar plots shown in Fig. $4-\mathrm{f}$. It is seen from Fig. $4 \mathrm{c}$ and $\mathrm{d}$ that the maxima of $\alpha_{0}$ and $\beta_{0}$ both occur when the incident light polarization is parallel to the armchair direction within an experimental error tolerance of approximately $3^{\circ}$. Considering the NA changes between different polarizations, we analyzed the proportion of 2PA in the NA by plotting the coefficient $k=\beta I /(\alpha+\beta I)$ in polar coordinates, as presented in Fig. $4 \mathrm{~g}$. It is found from Fig. $4 \mathrm{~g}$ that $2 \mathrm{PA}$ with higher $k$ at $90^{\circ}$ polarization angle takes a larger proportion compared with the $0^{\circ}$ case, indicating that the SA effect plays a crucial role in the NA process at $0^{\circ}$, but RSA dominates at $90^{\circ}$. These results are consistent with Fig. $4 a$ and $b$.

In order to vividly describe the polarization-dependent properties of the NA, the polarizations parallel to the armchair or zigzag direction are named as P1 or P2, respectively. There are three possible interband transitions when excited by $\mathrm{P} 1$ or $\mathrm{P} 2$, as illustrated in Fig. 2d. Transition types I, II, IV, and V belong to 1PA, which are included in the calculation of $\alpha_{0}$ and $I_{\mathrm{S}_{1}}$, while other two transitions, III and VI, are associated with 2PA contributing to $\beta_{0}$ and $I_{\mathrm{S}_{2}}$. Based on the results of the LA spectra mentioned above, for incident photon energy $\hbar \omega$ $=2.067 \mathrm{eV}$, transitions I, II, III, IV, and VI satisfy the law of energy conservation; however, the transition type $\mathrm{V}$ does not $\left(E_{1 \text {,Armchair } / 2}<E_{1, \text { Zigzag } / 2}<E_{\text {g,Armchair }}<E_{\text {g,Zigzag }}<\right.$ $\left.E_{1 \text {, Armchair }}<\hbar \omega<E_{1 \text {, Zigzag }}\right)$. In this case, the contribution of 2PA excited by $\mathrm{P} 1$ to the overall nonlinear signal is larger than that excited by P2. Consequently, the signals are dominated by SA (mainly caused by 1PA) effect at the P1 polarization, while RSA (mainly caused by 2PA) effect takes the main role at the $\mathrm{P} 2$ polarization, in agreement with Fig. $4 \mathrm{a}$ and $\mathrm{b}$. With regard to the incident beam with other polarizations, the electric field intensity can be projected onto the $\mathrm{P} 1$ and $\mathrm{P} 2$ directions, and their transitions are considered separately. From Fig. 2a, the pro- portion of small-signal 1PA, i.e., transitions I, II, and IV, can be deduced. If $\left(I_{586}-I_{614}\right) /\left(I_{586}+I_{614}\right)<0$, the transitions I and II are dominant, suggesting an SA response in NA. If $\left(I_{586}-I_{614}\right) /\left(I_{586}+I_{614}\right)>0$, the transition IV predominates, implying an RSA response. NA results validate our conjecture, as shown in Fig. 4g: $k=\beta I /(\alpha+\beta I)$ increases significantly when the polarization angle is between $21^{\circ}$ and $159^{\circ}$ and, thus, where the condition $\left(I_{586}-\right.$ $\left.I_{614}\right) /\left(I_{586}+I_{614}\right)>0$ is satisfied.

In this section, the different SA or RSA responses excited by P1 or P2 implies that the GeS/graphene heterostructure possesses a highly polarization-dependent NA property. This not only reflects its anisotropic electronic bandstructure but also provides great potentials for wide applications in polarization-controlled optoelectronic devices. Therefore, the next section furnishes a new perspective for all-optical switches based on the strong anisotropy of NA effect in the GeS/graphene heterostructure.

\section{Polarization-based all-optical switch by GeS flake}

To the best of our knowledge, there are mainly four types of switches [66]: the thermo-optic-based $[67,68]$, the optical-limiting-based [69,70], the plasmonic nanostructure-based [71-75] and the SA-based switches [57]. In this context, we will focus our study on the fourth category. It is known that $2 \mathrm{D}$-saturable absorbers are promising candidates for the high-performance passive Q-switched [76] and mode-locked [77] lasers, as well as all-optical switches and modulators [78,79]. Importantly, the realization of all-optical switches is primarily based on the nonlinear SA effect, which causes the transmittance change of materials [80]. As mentioned above, the GeS/ graphene heterostructure exhibits special polarizationdependent SA/RSA effect under a $600 \mathrm{~nm}$ femtosecond laser excitation. Therefore, we began to investigate the $\mathrm{GeS} /$ graphene heterostructure-based all-optical switch by considering its large modulation depth difference between SA and RSA effects. Notably, the nonlinear modulation type along armchair polarization corresponds to SA, which changes to RSA when the polarization direction of a $600 \mathrm{~nm}$ laser is parallel to zigzag. The schematic illustration of our experimental process is shown in Fig. $5 \mathrm{a}$. We employed a femtosecond laser ( $35 \mathrm{fs}, 600 \mathrm{~nm}$, $500 \mathrm{~Hz}, 12 \mathrm{GW} \mathrm{cm}^{-2}$ ) with P1 and P2 directions to switch "ON" and "OFF" states of the incident CW laser (532 nm, $80 \mathrm{nW}$ ) by altering the NA state of the GeS/graphene heterostructure. For instance, when the polarization of the incident femtosecond light with high power is along armchair, the transmittance of CW laser is in the high 
state representing “ON". The transmittance of CW laser switches to "OFF" state with polarization of a femtosecond laser changing to zigzag.

The conventional method of realizing all-optical switches is by altering transmittance of the modulated CW laser with the switching laser power increasing, as shown in Fig. 5b. In this figure, the transmittance of CW laser under a femtosecond laser along $0^{\circ}$ (armchair) and $90^{\circ}$ (zigzag) are plotted in red and gray dots, respectively. The relative values of the two polarizations are normalized based on red dots. More details are shown in the inserts of Fig. 5b, in which the modulated depths of the transmittance are $0.5 \%\left(0^{\circ}\right)$ and $-2.5 \%\left(90^{\circ}\right)$, respectively. In other words, the performance of the power-dependent all-optical switch is not high enough, since the transmittance difference cannot be distinguished at the selected polarization, no matter whether the light is $\mathrm{ON}$ or
OFF. However, as shown in Fig. $5 c$, the ON/OFF ratio increases when the state of $\mathrm{GeS} /$ graphene heterostructure changes from SA to RSA response. In Fig. 5, the vertical color bar in deep pink (light pink) represents the P1 (P2) polarization direction of femtosecond switching light. Here, P1/P2 can be chosen from three different groups: $0^{\circ} / 90^{\circ}$ (red line), $-10^{\circ} / 80^{\circ}$ (green line), $10^{\circ} / 100^{\circ}$ (blue line). Here, by comparing different modes, we found that the tunable modulation depth of $0^{\circ} / 90^{\circ}$ method is larger than those of the other polarization modes and ON/OFF modes. Particularly, the first group is along armchair and zigzag directions, while the other two are selected as control groups to verify the effect of all-optical switching.

Therefore, we established a novel all-optical switch utilizing the polarization-tunable SA/RSA effect of GeS/ graphene heterostructure with ON/OFF modes that can be switched by changing the polarization direction $(0 \%$
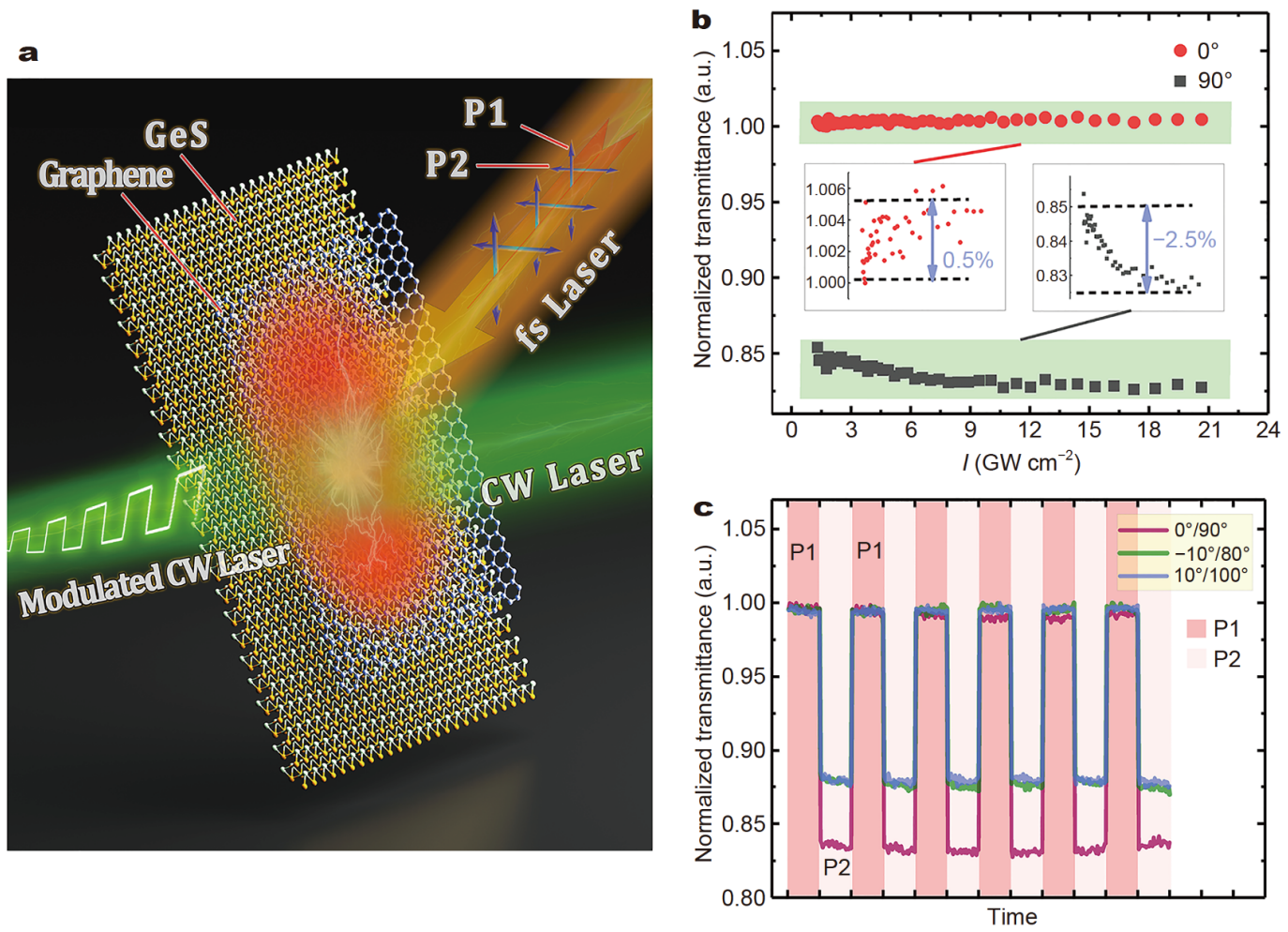

Figure 5 Sketch of the all-optical switch and normalized transmittance of the GeS/graphene switch. (a) Experimental process sketch of GeS/ graphene-based all-optical switch. The transmittance of the CW laser $(532 \mathrm{~nm}, 80 \mathrm{nW})$ was obtained with the polarization of switching femtosecond light ( 35 fs laser, $600 \mathrm{~nm}, 500 \mathrm{~Hz}, 12 \mathrm{GW} \mathrm{cm}^{-2}$ ) along P1 or P2 direction. The state of CW laser could be changed between "ON" and "OFF" back and forth through the GeS/graphene heterostructure. (b) Relative normalized transmittance with the increasing intensity of modulated CW laser along $0^{\circ}$ (armchair) and $90^{\circ}$ (zigzag). Relative values of the red curve and gray curve are not changed under the reference of the normalized red curve. From the insets of enlarged two curves at $20 \mathrm{GW} \mathrm{cm}{ }^{-2}$, the modulated depths of switched light come to $0.5 \%$ along $0^{\circ}$ and $-2.5 \%$ along $90^{\circ}$, respectively. (c) The normalized transmittance of the modulated CW light was obtained under constant-power switching femtosecond light of three different polarization modes: $0^{\circ} / 90^{\circ}$ (red line), $-10^{\circ} / 80^{\circ}$ (green line), $10^{\circ} / 100^{\circ}$ (blue line). Vertical color bars in deep pink and light pink represent polarization changing between P1 and P2 (P1/P2). Modulated light represents "ON" state under P1 polarization and "OFF" state under P2 polarization. Besides, the transmittances of both "ON" and "OFF" states are normalized under the reference of "ON" state in each case. 
$90^{\circ}$ ) of the switching femtosecond laser. This realization is attributed to the following two aspects. Firstly, GeS possesses a moderate direct bandgap that is irrelevant with thickness, and the nonlinear optical response is in the visible range. Secondly, owing to its anisotropic inplane structure, GeS special polarization-dependent bandgaps/absorption edges are used to generate polarization-tunable SA/RSA responses at a specific wavelength (i.e., $600 \mathrm{~nm}$ ). In the heterostructure, due to the narrow bandgap and isotropic structure, the origin of this anisotropy-optical switch phenomenon is the in-plane asymmetric structure of the GeS flake. Additionally, according to the optical transition selection rules, the polarization direction of a femtosecond laser would affect the absorption edges to which the electrons have been transferred. As a result, it is the polarized direction of femtosecond laser that determines the different transmittance intensities of the CW light. In other words, a high ON/OFF ratio of $17 \%(100 \%-83 \%)$ is achieved in the first polarization group $\left(0^{\circ} / 90^{\circ}\right)$ compared with the other two groups with the ON/OFF ratio of $12 \%$. Therefore, the "ON" and "OFF" modes can be realized and mostly optimized through this special method, in which we can adjust the polarization direction of switching laser between the armchair and zigzag to allow the GeS/graphene heterostructure to switch between the SA and RSA patterns.

\section{CONCLUSION}

In summary, we firstly conducted the polarization-dependent Raman spectroscopy to characterize the crystallographic axis orientation of $251 \mathrm{~nm}$ GeS flake with an anisotropic structure. In addition, the LA spectra were collected to determine the anisotropic $E_{1}$ energy bands of GeS flake in an indirect way. Our LA spectra results indicate that a $600 \mathrm{~nm}$ laser is a suitable choice for the investigation of polarization-dependent NA effects. Importantly, an obvious SA effect $(\delta T>0)$ was observed along armchair, whereas an RSA effect $(\delta T<0)$ was found along zigzag. These two effects co-exist in cases of polarization angles between armchair and zigzag. Additionally, it was confirmed that the transmittance of CW laser could be modulated by controlling its polarization direction of a $600 \mathrm{~nm}$ femtosecond switching laser injected on the GeS/graphene heterostructure, in which the "ON" and "OFF" states can be accessed by changing the NA patterns (SA/RSA). This work provides significant implications for the development of the next-generation all-optical switches/modulators and other advanced photonic devices.
Received 6 January 2020; accepted 7 March 2020; published online 22 April 2020

1 Novoselov KS, Geim AK, Morozov SV, et al. Electric field effect in atomically thin carbon films. Science, 2004, 306: 666-669

2 Novoselov KS, Geim AK, Morozov SV, et al. Two-dimensional GaS of massless Dirac fermions in graphene. Nature, 2005, 438: 197-200

3 Jiang T, Huang D, Cheng J, et al. Gate-tunable third-order nonlinear optical response of massless Dirac fermions in graphene. Nat Photon, 2005, 12: 430-436

4 Zhang J, Ouyang $\mathrm{H}$, Zheng $\mathrm{X}$, et al. Ultrafast saturable absorption of $\mathrm{MoS}_{2}$ nanosheets under different pulse-width excitation conditions. Opt Lett, 2018, 43: 243-246

5 Yang $\mathrm{X}, \mathrm{Li} \mathrm{Q}, \mathrm{Hu} \mathrm{G}$, et al. Controlled synthesis of high-quality crystals of monolayer $\mathrm{MoS}_{2}$ for nanoelectronic device application. Sci China Mater, 2016, 59: 182-190

6 Zhang S, Dong N, McEvoy N, et al. Direct observation of degenerate two-photon absorption and its saturation in $\mathrm{WS}_{2}$ and $\mathrm{MoS}_{2}$ monolayer and few-layer films. ACS Nano, 2015, 9: 7142-7150

7 Zheng $\mathrm{C}, \mathrm{Xu} \mathrm{ZQ}$, Zhang Q, et al. Profound effect of substrate hydroxylation and hydration on electronic and optical properties of monolayer $\mathrm{MoS}_{2}$. Nano Lett, 2015, 15: 3096-3102

8 Saynatjoki A, Karvonen L, Rostami $\mathrm{H}$, et al. Ultra-strong nonlinear optical processes and trigonal warping in $\mathrm{MoS}_{2}$ layers. Nat Commun, 2017, 8: 893

9 Hong S, Fu D, Hou J, et al. Robust photoluminescence energy of $\mathrm{MoS}_{2}$ /graphene heterostructure against electron irradiation. Sci China Mater, 2018, 61: 1351-1359

10 Sobota JA, Yang SL, Kemper AF, et al. Direct optical coupling to an unoccupied Dirac surface state in the topological insulator $\mathrm{Bi}_{2}$ $\mathrm{Se}_{3}$. Phys Rev Lett, 2013, 111: 136802

11 Zhang $\mathrm{H}$, Liu CX, Qi XL, et al. Topological insulators in $\mathrm{Bi}_{2} \mathrm{Se}_{3}$, $\mathrm{Bi}_{2} \mathrm{Te}_{3}$ and $\mathrm{Sb}_{2} \mathrm{Te}_{3}$ with a single Dirac cone on the surface. Nat Phys, 2013, 5: 438-442

12 Zhang J, Jiang T, Zhou T, et al. Saturated absorption of different layered $\mathrm{Bi}_{2} \mathrm{Se}_{3}$ films in the resonance zone. Photon Res, 2018, 6: C8

13 Jiang T, Miao RL, Zhao J, et al. Electron-phonon coupling in topological insulator $\mathrm{Bi}_{2} \mathrm{Se}_{3}$ thin films with different substrates. Chin Opt Lett, 2019, 17: 020005

14 Meng X, Zhou Y, Chen K, et al. Anisotropic saturable and excitedstate absorption in bulk $\operatorname{ReS}_{2}$. Adv Opt Mater, 2018, 6: 1800137

15 Li XL, Han WP, Wu JB, et al. Layer-number dependent optical properties of 2D materials and their application for thickness determination. Adv Funct Mater, 2017, 27: 1604468

16 Guo B, Xiao Q, Wang S, et al. 2D layered materials: Synthesis, nonlinear optical properties, and device applications. Laser Photonics Rev, 2019, 13: 1800327

17 Jiang $\mathrm{T}$, Yin $\mathrm{K}$, Wang C, et al. Ultrafast fiber lasers mode-locked by two-dimensional materials: Review and prospect. Photon Res, 2020, 8: 78

18 Guo Z, Zhang H, Lu S, et al. From black phosphorus to phosphorene: Basic solvent exfoliation, evolution of Raman scattering, and applications to ultrafast photonics. Adv Funct Mater, 2015, 25: 6996-7002

19 Yang T, Abdelwahab I, Lin $\mathrm{H}$, et al. Anisotropic third-order nonlinearity in pristine and lithium hydride intercalated black phosphorus. ACS Photonics, 2018, 5: 4969-4977

20 Zhou Y, Zhang M, Guo Z, et al. Recent advances in black phosphorus-based photonics, electronics, sensors and energy devices. 
Mater Horiz, 2017, 4: 997-1019

21 Liu E, Fu Y, Wang Y, et al. Integrated digital inverters based on two-dimensional anisotropic $\mathrm{ReS}_{2}$ field-effect transistors. Nat Commun, 2015, 6: 6991

22 Liu F, Zheng S, He X, et al. Highly sensitive detection of polarized light using anisotropic 2D $\mathrm{ReS}_{2}$. Adv Funct Mater, 2016, 26: 11691177

23 Huang S, Tatsumi Y, Ling X, et al. In-plane optical anisotropy of layered gallium telluride. ACS Nano, 2016, 10: 8964-8972

24 Luo Z, Maassen J, Deng Y, et al. Anisotropic in-plane thermal conductivity observed in few-layer black phosphorus. Nat Commun, 2015, 6: 8572

25 Youngblood N, Peng R, Nemilentsau A, et al. Layer-tunable thirdharmonic generation in multilayer black phosphorus. ACS Photonics, 2016, 4: 8-14

26 Yang S, Liu Y, Wu M, et al. Highly-anisotropic optical and electrical properties in layered SnSe. Nano Res, 2017, 11: 554-564

27 Ye Y, Xian Y, Cai J, et al. Linear and nonlinear optical properties of few-layer exfoliated SnSe nanosheets. Adv Opt Mater, 2019, 7: 1800579

28 Xia F, Wang H, Jia Y. Rediscovering black phosphorus as an anisotropic layered material for optoelectronics and electronics. Nat Commun, 2014, 5: 4458

29 Li L, Yu Y, Ye GJ, et al. Black phosphorus field-effect transistors. Nat Nanotech, 2014, 9: 372-377

30 Wei Y, He J, Zhang Q, et al. Synthesis and investigation of layered $\mathrm{GeS}$ as a promising large capacity anode with low voltage and high efficiency in full-cell Li-ion batteries. Mater Chem Front, 2017, 1: 1607-1614

31 Hu X, Huang P, Liu K, et al. Salt-assisted growth of ultrathin GeSe rectangular flakes for phototransistors with ultrahigh responsivity. ACS Appl Mater Interfaces, 2019, 11: 23353-23360

32 Zhou X, $\mathrm{Hu} \mathrm{X}$, Jin B, et al. Highly anisotropic GeSe nanosheets for phototransistors with ultrahigh photoresponsivity. Adv Sci, 2018, 5: 1800478

33 Wu L, Xie Z, Lu L, et al. Few-layer tin sulfide: A promising blackphosphorus-analogue 2D material with exceptionally large nonlinear optical response, high stability, and applications in all-optical switching and wavelength conversion. Adv Opt Mater, 2018, 6: 1700985

$34 \mathrm{Xu} \mathrm{X}$, Song Q, Wang $\mathrm{H}$, et al. In-plane anisotropies of polarized Raman response and electrical conductivity in layered tin selenide. ACS Appl Mater Interfaces, 2017, 9: 12601-12607

35 Zhou X, Hu X, Zhou S, et al. Tunneling diode based on $\mathrm{WSe}_{2} / \mathrm{SnS}_{2}$ heterostructure incorporating high detectivity and responsivity. Adv Mater, 2018, 30: 1703286

36 Zhang S, Liu S, Huang S, et al. Structural and electronic properties of atomically thin germanium selenide polymorphs. Sci China Mater, 2015, 58: 929-935

37 Qin G, Qin Z, Fang WZ, et al. Diverse anisotropy of phonon transport in two-dimensional group IV-VI compounds: A comparative study. Nanoscale, 2016, 8: 11306-11319

38 Shafique A, Shin YH. Thermoelectric and phonon transport properties of two-dimensional IV-VI compounds. Sci Rep, 2017, 7: 506

39 Hsueh HC, Li JX, Ho CH. Polarization photoelectric conversion in layered GeS. Adv Opt Mater, 2018, 6: 1701194

40 Li F, Liu X, Wang Y, et al. Germanium monosulfide monolayer: A novel two-dimensional semiconductor with a high carrier mobility. J Mater Chem C, 2016, 4: 2155-2159
41 Zhang S, Wang N, Liu S, et al. Two-dimensional GeS with tunable electronic properties via external electric field and strain. Nanotechnology, 2016, 27: 274001

42 Guo SD, Wang YH. Thermoelectric properties of orthorhombic group IV-VI monolayers from the first-principles calculations. J Appl Phys, 2017, 121: 034302

43 Ma Z, Wang B, Ou L, et al. Structure and properties of phosphorene-like IV-VI 2D materials. Nanotechnology, 2016, 27: 415203

44 Li BW, Wang Y, Xie YQ, et al. Strain controlled switching effects in phosphorene and GeS. Nanotechnology, 2017, 28: 435202

45 Tan D, Lim HE, Wang F, et al. Anisotropic optical and electronic properties of two-dimensional layered germanium sulfide. Nano Res, 2016, 10: 546-555

46 Ho $\mathrm{CH}$, Li JX. Polarized band-edge emission and dichroic optical behavior in thin multilayer GeS. Adv Opt Mater, 2017, 5: 1600814

47 Ulaganathan RK, Lu YY, Kuo CJ, et al. High photosensitivity and broad spectral response of multi-layered germanium sulfide transistors. Nanoscale, 2016, 8: 2284-2292

48 Sheliakina M, Mostert AB, Meredith P. An all-solid-state biocompatible ion-to-electron transducer for bioelectronics. Mater Horiz, 2018, 5: 256-263

49 Huang L, Wu F, Li J. Structural anisotropy results in strain-tunable electronic and optical properties in monolayer GeX and $\mathrm{SnX}$ (X = S, Se, Te). J Chem Phys, 2016, 144: 114708

50 Gomes LC, Carvalho A. Phosphorene analogues: Isoelectronic twodimensional group-IV monochalcogenides with orthorhombic structure. Phys Rev B, 2015, 92: 085406

51 Gomes LC, Trevisanutto PE, Carvalho A, et al. Strongly bound Mott-Wannier excitons in GeS and GeSe monolayers. Phys Rev B, 2016, 94: 155428

52 Ju L, Dai Y, Wei W, et al. DFT investigation on two-dimensional $\mathrm{GeS} \mathrm{WS}_{2}$ van der Waals heterostructure for direct Z-scheme photocatalytic overall water splitting. Appl Surf Sci, 2018, 434: 365-374

53 Cheng $\mathrm{K}$, Guo $\mathrm{Y}$, Han $\mathrm{N}$, et al. Lateral heterostructures of monolayer group-IV monochalcogenides: Band alignment and electronic properties. J Mater Chem C, 2017, 5: 3788-3795

54 Gomes LC, Carvalho A, Castro Neto AH. Vacancies and oxidation of two-dimensional group-IV monochalcogenides. Phys Rev B, 2016, 94: 054103

55 Wang S, Yu H, Zhang $\mathrm{H}$, et al. Broadband few-layer $\mathrm{MoS}_{2}$ saturable absorbers. Adv Mater, 2014, 26: 3538-3544

56 Ferrari AC, Basko DM. Raman spectroscopy as a versatile tool for studying the properties of graphene. Nat Nanotech, 2013, 8: 235246

57 Zhang C, Ouyang H, Miao R, et al. Anisotropic nonlinear optical properties of a SnSe flake and a novel perspective for the application of all-optical switching. Adv Opt Mater, 2019, 7: 1900631

58 Fadil D, Hossain RF, Saenz GA, et al. On the chemically-assisted excitonic enhancement in environmentally-friendly solution dispersions of two-dimensional $\mathrm{MoS}_{2}$ and $\mathrm{WS}_{2}$. J Mater Chem C, 2017, 5: 5323-5333

59 Chaguetmi S, Mammeri F, Nowak S, et al. Photocatalytic activity of $\mathrm{TiO}_{2}$ nanofibers sensitized with $\mathrm{ZnS}$ quantum dots. RSC Adv, 2013, 3: 2572-2580

60 Ling X, Huang S, Hasdeo EH, et al. Anisotropic electron-photon and electron-phonon interactions in black phosphorus. Nano Lett, 2016, 16: 2260-2267

61 Ul Haq B, AlFaify S, Laref A, et al. Optoelectronic properties of 
new direct bandgap polymorphs of single-layered germanium sulfide. Ceramics Int, 2019, 45: 18073-18078

62 Balandin AA, Ghosh S, Bao W, et al. Superior thermal conductivity of single-layer graphene. Nano Lett, 2008, 8: 902-907

63 Sheik-Bahae M, Said AA, Wei TH, et al. Sensitive measurement of optical nonlinearities using a single beam. IEEE J Quantum Electron, 1990, 26: 760-769

64 Chen R, Zheng X, Jiang T. Broadband ultrafast NA and ultra-long exciton relaxation time of black phosphorus quantum dots. Opt Express, 2017, 25: 7507-7519

65 Zhang J, Jiang T, Zheng X, et al. Thickness-dependent nonlinear optical properties of $\mathrm{CsPbBr}_{3}$ perovskite nanosheets. Opt Lett, 2017, 42: 3371-3374

66 Chen H, Wang C, Ouyang H, et al. All-optical modulation with 2D layered materials: Status and prospects. Nanophotonics, 2020

67 Wu K, Wang Y, Qiu C, et al. Thermo-optic all-optical devices based on two-dimensional materials. Photon Res, 2018, 6: C22

$68 \mathrm{Wu} \mathrm{K}$, Guo C, Wang H, et al. All-optical phase shifter and switch near $1550 \mathrm{~nm}$ using tungsten disulfide $\left(\mathrm{WS}_{2}\right)$ deposited tapered fiber. Opt Express, 2017, 25: 17639-17649

69 Chen Y, Bai T, Dong N, et al. Graphene and its derivatives for laser protection. Prog Mater Sci, 2016, 84: 118-157

70 Dong N, Li Y, Feng Y, et al. Optical limiting and theoretical modelling of layered transition metal dichalcogenide nanosheets. Sci Rep, 2015, 5: 14646

71 Pasquazi A, Stivala S, Assanto G, et al. In situ tuning of a photonic band gap with laser pulses. Appl Phys Lett, 2008, 93: 091111

72 Dani KM, Ku Z, Upadhya PC, et al. Subpicosecond optical switching with a negative index metamaterial. Nano Lett, 2009, 9: 3565-3569

73 Miao $\mathrm{R}, \mathrm{Hu} \mathrm{Y}$, Ouyang $\mathrm{H}$, et al. A polarized nonlinear optical response in a topological insulator $\mathrm{Bi}_{2} \mathrm{Se}_{3}-\mathrm{Au}$ nanoantenna hybridstructure for all-optical switching. Nanoscale, 2019, 11: 1459814606

$74 \mathrm{Hu} \mathrm{Y}$, Jiang $\mathrm{T}$, Zhou J, et al. Ultrafast terahertz frequency and phase tuning by all-optical molecularization of metasurfaces. Adv Opt Mater, 2019, 7: 1901050

75 Hu Y, Jiang T, Zhou J, et al. Ultrafast terahertz transmission/group delay switching in photoactive $\mathrm{WSe}_{2}$-functionalized metaphotonic devices. Nano Energy, 2020, 68: 104280

76 Bao Q, Zhang H, Wang Y, et al. Atomic-layer graphene as a saturable absorber for ultrafast pulsed lasers. Adv Funct Mater, 2009, 19: 3077-3083

77 Baek IH, Lee HW, Bae S, et al. Efficient mode-locking of sub-70-fs Ti:sapphire laser by graphene saturable absorber. Appl Phys Express, 2012, 5: 032701

78 Wu L, Jiang X, Zhao J, et al. MXene-based nonlinear optical information converter for all-optical modulator and switcher. Laser Photonics Rev, 2018, 12: 1800215

79 Ma D, Zhao J, Wang R, et al. Ultrathin GeSe nanosheets: From systematic synthesis to studies of carrier dynamics and applications for a high-performance UV-VIS photodetector. ACS Appl Mater Interfaces, 2019, 11: 4278-4287

80 Chai Z, Hu X, Wang F, et al. Ultrafast all-optical switching. Adv Opt Mater, 2016, 5: 1600665

Acknowledgements The authors are grateful for financial support from the National Natural Science Foundation of China (11802339, 11805276, 61805282, 61801498, 11804387, and 11902358), the Scientific Researches Foundation of National University of Defense Technology (ZK16-03-59, ZK18-01-03, ZK18-03-36, and ZK18-03-22), the Natural Science Foundation of Hunan province (2016JJ1021), the Open Director Fund of State Key Laboratory of Pulsed Power Laser Technology (SKL2018ZR05), the Open Research Fund of Hunan Provincial Key Laboratory of High Energy Technology (GNJGJS03), the Opening Foundation of State Key Laboratory of Laser Interaction with Matter (SKLLIM1702), and the Youth Talent Lifting Project (17-JCJQ-QT004).

Author contributions Jiang $\mathrm{T}$ proposed and designed the project. Ouyang $\mathrm{H}$, Zhang $\mathrm{C}$ and Liu Q contributed equally to this work on fabricating the samples, conducting the experiments, analyzing the data and writing the paper. Hu S, Zhang J, Hao H, You J and Cheng X participated in the experiments, writing the paper and revising the manuscript. All authors contributed to the general discussion.

Conflict of interest The authors declare that they have no conflict of interest. 


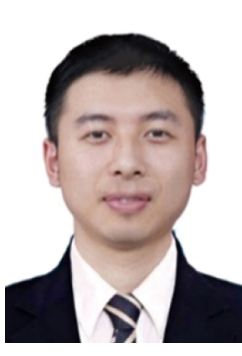

Hao Ouyang received his BSc degree from the National University of Defence Technology in 2018. Currently, he is a graduate student in the group of Prof. Tian Jiang at the National University of Defence Technology. His research interest mainly focuses on nonlinear optic laser and materials interaction.

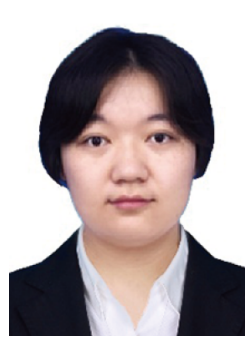

Chenxi Zhang is a postgraduate student under the guidance of Prof. Tian Jiang at the National University of Defense Technology. She received her BSc degree in photoelectric information science and engineering from the University of Science and Technology of China in 2018. Her current research focuses on the optical nonlinear properties of $2 \mathrm{D}$ materials and their applications in all-optical devices.

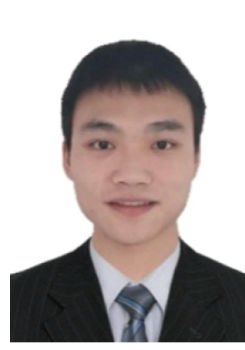

Qirui Liu received his BSc degree from Army Engineering University in 2018. Currently, he is a graduate student in the group of Prof. Tian Jiang at the National University of Defence Technology. His research interest concentrates on the field of nonlinear optics and ultrafast spectroscopy.

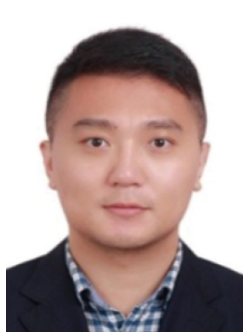

Tian Jiang is a professor at the College of Advanced Interdisciplinary Studies, National University of Defense Technology, Changsha, China. He is a well-known researcher in the field of light-matter interactions in condensed matter physics. His research interests include topological insulators, ultrafast dynamics of energy relaxation, spintronics, and radiative recombination of quasi-particles in transition metal dichalcogenides (TMDCs), black phosphorus and other low-dimensional materials.
各向异性硫化锗薄片从饱和吸收到反饱和吸收的 偏振可调非线性吸收模式及其在全光开关中的应 用

欧阳吴 ${ }^{1,2,3 \dagger}$, 张晨希 ${ }^{1 \dagger}$, 刘祺瑞 ${ }^{1 \dagger}$, 胡思扬 ${ }^{1}$, 张峻 ${ }^{4}$, 郝昊 $^{4}, \mathrm{e}^{\mathrm{C}^{*}}{ }^{5}$,

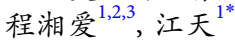

摘要 近年来, 一些二维层状材料, 尤其是具有褶皱蜂窝结构的IVVI族单硫化物, 由于其特殊的各向异性化学和物理性质, 而备受关 注. 在IV-VI族层状化合物 MX ( $\mathrm{M}=\mathrm{Ge}, \mathrm{Sn} ; \mathrm{X}=\mathrm{Se}, \mathrm{S})$ 中, 硫化锗 $(\mathrm{GeS})$ 以其最强的各向异性热导率和热电品质因子而引人注目. 此外, $\mathrm{GeS}$ 薄片与层数无关的直接能带 $\left(E_{\mathrm{g}} \sim 1.6 \mathrm{eV}, E_{1} \sim 2.1 \mathrm{eV}\right)$ 为可见光光 探测器的应用提供了良好的应用前景. 本文研究了 GeS薄片的偏振 可调非线性吸收模式, 利用偏振相关拉曼光谱和线性吸收光谱表 征了厚度为 $251 \mathrm{~nm} \mathrm{GeS}$ 的晶格取向和吸收能带. 考虑到 $\mathrm{GeS}$ 薄片的 较低的热损伤阈值，在不改变 GeS 非线性特性的前提下，制备了硫 化锗/石墨烯异质结. 非线性吸收结果表明, 在硫化锗/石墨烯异质 结中, 利用偏振可调的 $600 \mathrm{~nm}$ 线偏振飞秒激光沿扶手椅方向激发 产生饱和吸收(SA)效应, 沿锯齿方向激发反饱和吸收(RSA)效应. 实验验证了基于偏振的硫化锗/石墨烯异质结全光开关的功能. 值 得注意的是, 正因为 $\mathrm{GeS}$ 的偏振相关非线性吸收模式(SA/RSA), 全 光开关的“开”、“关”状态可通过控制飞秒开关激光 $(600 \mathrm{~nm}, 35 \mathrm{fs}$, $\left.500 \mathrm{~Hz}, 12 \mathrm{GW} \mathrm{cm}^{-2}\right)$ 的偏振, 进而改变连续光 $(\mathrm{CW})$ 激光 $(532 \mathrm{~nm}$, $80 \mathrm{nW})$ 透射率的方式来实现. 通过改变开关光的偏振, 开关比可以 达到 $17 \%$, 与之相比, 通过提高开关光入射功率获得的开关比仅为 3.0\%. 本文介绍的偏振可调吸收模式, 为基于硫化锗/石墨烯异质结 的下一代光电子器件开辟了新的前景. 